\title{
A Note on the Formulas for the Drazin Inverse of the Sum of Two Matrices and Its Applications
}

\author{
Xiaolan Qin*, Ricai Luo \\ School of Mathematics and Statistics, Hechi University, Yizhou, China \\ Email address: \\ qinxiaolan18@163.com (Xiaolan Qin), luoricai@163.com (Ricai Luo) \\ ${ }^{*}$ Corresponding author
}

\section{To cite this article:}

Xiaolan Qin, Ricai Luo. A Note on the Formulas for the Drazin Inverse of the Sum of Two Matrices and Its Applications. Pure and Applied Mathematics Journal. Vol. 8, No. 3, 2019, pp. 54-71. doi: 10.11648/j.pamj.20190803.12

Received: July 6, 2019; Accepted: August 10, 2019; Published: August 30, 2019

\begin{abstract}
The Drazin inverse has applications in a number of areas such as control theory, Markov chains, singular differential and difference equations, and iterative methods in numerical linear algebra. The study on representations for the Drazin inverse of block matrices stems essentially from finding the general expressions for the solutions to singular systems of differential equations, and then stimulated by a problem formulated by Campbell. In 1983, Campbell (Campbell et al. (1976)) established an explicit representation for the Drazin inverse of a $2 \times 2$ block matrix $\mathrm{M}$ in terms of the blocks of the partition, where the blocks A and D are assumed to be square matrices. Special cases of the problems have been studied. In 2009, Chunyuan Deng and Yimin Wei found an explicit representation for the Drazin inverse of an anti-triangular matrix M, where A and $\mathrm{BC}$ are generalized Drazin invertible, if $\mathrm{A}^{\pi} \mathrm{AB}=0$ and $\mathrm{BC}\left(\mathrm{I}-\mathrm{A}^{\pi}\right)=0$. Afterwards, several authors have investigated this problem under some limited conditions on the blocks of $\mathrm{M}$. In particular, a representation of the Drazin inverse of M, denoted by $\mathrm{M}^{\mathrm{d}}$. In this paper, we consider the Drazin inverse of a sum of two matrices and we derive additive formulas under the conditions of $\mathrm{ABA}^{\pi}=0$ and $\mathrm{BA}^{\pi}=0$ respectively. Precisely, for a block matrix $\mathrm{M}$, we give a new representation of $\mathrm{M}^{\mathrm{d}}$ under some conditions that $\mathrm{AB}=0$ and $\mathrm{DCA}^{\pi}=0$. Moreover, some particular cases of this result related to the Drazin inverse of block matrices are also considered.
\end{abstract}

Keywords: Drazin Inverse, Block Matrices, Drazin Index

\section{Introduction}

Let $\mathrm{A}$ be a square complex matrix. We denote by $\mathrm{R}(\mathrm{A}), \mathrm{N}$ (A) and rank (A), the range, the null space and the rank of matrix $\mathrm{A}$, respectively. In addition, the smallest integer $\mathrm{k}$ is called the Drazin index of A such that rank $\left(A^{k+1}\right)=$ rank $\left(A^{k}\right)$. Let $\mathbb{C}^{n \times n}$ be the set of all the $n \times n$ matrices over the complex field, for every matrix $A \in \mathbb{C}^{n \times n}$, such that $\operatorname{index}(A)=k$, there exists an unique matrix $A^{d} \in \mathbb{C}^{n \times n}$, which satisfies the relations:

$$
A^{d} A A^{d}=A^{d}, A^{d} A=A A^{d}, A^{k+1} A^{d}=A^{k} .
$$

The matrix $A^{d}$ is called the Drazin inverse of $A[1,2]$. The case $\operatorname{index}(A)=0$ is valid if and only if $A$ is nonsingular, so $A^{d}$ reduces to $A^{-1}$. By $A^{\pi}=I-A A^{d}$, we denote the projection on $N\left(A^{k}\right)$ along $R\left(A^{k}\right)$. If the lower limit of a sum is greater than its upper limit, we always define the sum to be 0 . For example, the sum $\sum_{l=1}^{k-2} *=0$ for $k \leq 2$. We agree that $A^{0}=I$, for any matrix $A$.

The study on representations for the Drazin inverse of block matrices essentially originated from finding the general expressions for the solutions to singular systems of differential equations [3-5], and then stimulated by a problem formulated by Campbell [3]: establish an explicit representation for the Drazin inverse of $2 \times 2$ block matrices

$$
M=\left[\begin{array}{ll}
A & B \\
C & D
\end{array}\right],
$$

in terms of the blocks of the partition, where the blocks A and $\mathrm{D}$ are assumed to be square matrices. For a deeper discussion 
of applications of the Drazin inverse of a $2 \times 2$ block matrix, we refer the reader to [5, 6]. Meyer and Rose [7], and independently Hartwig and Shoaf [8], first gave the formulas for block triangular matrices. Several authors have investigated this problem and they were able to find some partial answers (imposing some conditions on the blocks of $\mathrm{M})$. Here, we list some cases of Drazin inverse of block matrix M:

(1) $A B=0$ and $D C=0$. See [9].

(2) $B C=0$ and $B D=0$. See [10].

(3) $B C=0$ and $D C=0$. See [11].

(4) $A A^{\pi} B=0, B C A A^{d}=0$ and $\mathrm{DC}=0$. See [12]

(5) $B C A=0, B C B=0, D C A=0$ and $D C B=0$. See [13].

(6) $B C A=0, C B D=0, A(B C)^{\pi}=0$ and $D(C B)^{\pi}=0$.

See [14].

The motivation for this article is [15]. In the paper, the authors considered some conditions on $\mathrm{a}, \mathrm{b} \in \Lambda$ ( Let $\Lambda$ be a complex Banach algebra with the unit 1$)$ that allowed them to express $(a+b) d$ in terms of $a, a d, b$, bd. In this paper, we consider the Drazin inverse of a sum of two matrices and we derive additive formulas under the conditions of $\mathrm{ABA} \pi=0$ and $\mathrm{BA} \pi=0$ respectively. As an application we give some new representations for the Drazin inverse of a block matrix.

\section{A New Additive Result for the Drazin Inverse of Matrices}

First, we will state some auxiliary lemmas.

Lemma 2.1 [16] Let $A, B \in \mathbb{C}^{n \times n}$. If $A B=0$ an $A$ is nilpotent, then

$$
(A+B)^{d}=\sum_{i=0}^{r-1}\left(B^{d}\right)^{i+1} A^{i}, \quad \text { ind }(A)=r
$$

Lemma 2.2 [7] Let $M_{1}$ and $M_{2}$ be complex matrices of the form

$$
M_{1}=\left[\begin{array}{ll}
A & 0 \\
C & B
\end{array}\right], \quad M_{2}=\left[\begin{array}{ll}
B & C \\
0 & A
\end{array}\right],
$$

where $A$ and $B$ are complex square matrices. Let $r=\operatorname{ind}(A)$ and $s=\operatorname{ind}(B)$. Then $\max \{r, s\} \leq$ $\operatorname{ind}\left(M_{i}\right) \leq r+s$, for $i=1,2$, and

$$
\begin{aligned}
M_{1}^{d}=\left[\begin{array}{cc}
A^{d} & 0 \\
S & B^{d}
\end{array}\right], M_{2}^{d}=\left[\begin{array}{cc}
B^{d} & S \\
0 & A^{d}
\end{array}\right], \\
0=A B A^{\pi}=P\left[\begin{array}{cc}
A_{1} & 0 \\
0 & A_{2}
\end{array}\right]\left[\begin{array}{ll}
B_{1} & B_{2} \\
B_{3} & B_{4}
\end{array}\right]\left[\begin{array}{ll}
0 & 0 \\
0 & I
\end{array}\right] P^{-1}=P\left[\begin{array}{ll}
0 & A_{1} B_{2} \\
0 & A_{2} B_{4}
\end{array}\right] P^{-1} .
\end{aligned}
$$

From $A B A^{\pi}=0$, we have

$$
\begin{aligned}
& \text { where } \\
& \qquad S=\left(B^{d}\right)^{2} \sum_{i=0}^{r-1}\left(B^{d}\right)^{i} C A^{i} A^{\pi}+B^{\pi} \sum_{i=0}^{S-1} B^{i} C\left(A^{d}\right)^{i}\left(A^{d}\right)^{2}-B^{d} C A^{d} .
\end{aligned}
$$

The following theorem, we obtain the same expression for the Drazin inverse $(A+B)^{d}$ as in [15, Theorem2.3] for the Generalized Drazin inverse in a Banach Algebra.

Theorem 2.1. Let $A, B \in \mathbb{C}^{n \times n}$. If $A B A^{\pi}=0$, we have

$$
\begin{aligned}
&(A+B)^{d}= W^{d}+\sum_{i=0}^{r-1}\left(B^{d}\right)^{i+1} A^{i} A^{\pi}-\sum_{i=0}^{r-1}\left(B^{d}\right)^{i+1} A^{i} A^{\pi} B W^{d} \\
&+\sum_{j=0}^{k-1}\left(\sum_{i=0}^{r-1}\left(B^{d}\right)^{i+j+2} A^{i}\right) A^{\pi} B W^{j} W^{\pi} \\
&+B^{\pi} \sum_{j=0}^{k-1}(A+B)^{j} A^{\pi} B\left(W^{d}\right)^{j+2} \\
&-\sum_{j=0}^{k-1}\left(\sum_{i=0}^{r-1}\left(B^{d}\right)^{i+1} A^{i+1}\right)(A+B)^{j} A^{\pi} B\left(W^{d}\right)^{j+2}
\end{aligned}
$$

where $W=A A^{d}(A+B)$ and $\operatorname{ind}(A)=r, \quad$ ind $(B)=t$, $\max \{\operatorname{ind}(A), \operatorname{ind}(B)\} \quad \leq k \leq \operatorname{ind}(A)+\operatorname{ind}(B), \quad \operatorname{ind}(W) \leq$ $\operatorname{ind}(A)+\operatorname{ind}(B)$.

Proof. If we represent

$$
A \text { as } A=P\left[\begin{array}{cc}
A_{1} & 0 \\
0 & A_{2}
\end{array}\right] P^{-1},
$$

where $P$ and $A_{1}$ are nonsingular and $A_{2}$ is nilpotent, then

$$
A^{d}=P\left[\begin{array}{rr}
A_{1}^{-1} & 0 \\
0 & 0
\end{array}\right] P^{-1}
$$

and $\operatorname{ind}(A)=\operatorname{ind}\left(A_{1}\right)$. Let us write

$$
B=P\left[\begin{array}{ll}
B_{1} & B_{2} \\
B_{3} & B_{4}
\end{array}\right] P^{-1},
$$

where $B_{1} \in \mathbb{C}^{r \times r}$ being $r$ the size of $A_{1}$.

Therefore $A_{1} B_{2}=0$ and $A_{2} B_{4}=0$. The nonsingular of $A_{1}$ leads to $B_{2}=0$. Hence, 


$$
B=P\left[\begin{array}{cc}
B_{1} & 0 \\
B_{3} & B_{4}
\end{array}\right] P^{-1}, A+B=P\left[\begin{array}{cc}
A_{1}+B_{1} & 0 \\
B_{3} & A_{2}+B_{4}
\end{array}\right] P^{-1}
$$

Observe that

$$
W=A A^{d}(A+B)=P\left[\begin{array}{cc}
A_{1} & 0 \\
0 & A_{2}
\end{array}\right]\left[\begin{array}{cc}
A_{1}^{-1} & 0 \\
0 & 0
\end{array}\right]\left[\begin{array}{cc}
A_{1}+B_{1} & 0 \\
B_{3} & A_{2}
\end{array}\right] P^{-1}=P\left[\begin{array}{cc}
A_{1}+B_{1} & 0 \\
0 & 0
\end{array}\right] P^{-1},
$$

which leads to

$$
W^{d}=P\left[\begin{array}{cc}
\left(A_{1}+B_{1}\right)^{d} & 0 \\
0 & 0
\end{array}\right] P^{-1}
$$

Since $A_{2}$ is nilpotent and $A_{2} B_{4}=0$, Lemma 2.1 yields

$$
\left(A_{2}+B_{4}\right)^{d}=\sum_{i=0}^{r-1}\left(B_{4}^{d}\right)^{i+1} A_{2}^{i} .
$$

Thus, by lemma 2.2 , we obtain

$$
(A+B)^{d}=P\left[\begin{array}{cc}
\left(A_{1}+B_{1}\right)^{d} & 0 \\
S & \left(A_{2}+B_{4}\right)^{d}
\end{array}\right] P^{-1}
$$

and

$$
S=\sum_{j=0}^{p-1}\left(\left(A_{2}+B_{4}\right)^{d}\right)^{j+2} B_{3}\left(A_{1}+B_{1}\right)^{j}\left(A_{1}+B_{1}\right)^{\pi}+\sum_{j=0}^{q-1}\left(A_{2}+B_{4}\right)^{\pi}\left(A_{2}+B_{4}\right)^{j} B_{3}\left(\left(A_{1}+B_{1}\right)^{d}\right)^{j+2}-\left(A_{2}+B_{4}\right)^{d} B_{3}\left(A_{1}+B_{1}\right)^{d},
$$

where $p=\operatorname{ind}\left(A_{1}+B_{1}\right)$ and $q=\operatorname{ind}\left(A_{2}+B_{2}\right)$. Observe that for any $X \in \mathbb{C}^{m \times m}$, one has ind $(X) \leq m$ and if $k \geq \operatorname{ind}(X)$, then $X^{k} X^{\pi}=0$ (both affirmations can be proved by means of the Jordan canonical form of $X$ ). Thus, in (4) the upper limits of the summations can be replaced by simply $n$.

We have (we will write with an asterisk any entry whose exactly expression is not necessary)

$$
\left(B^{d}\right)^{i+1} A^{i} A^{\pi}=P\left[\begin{array}{cc}
\left(B_{1}^{d}\right)^{i+1} & 0 \\
* & \left(B_{4}^{d}\right)^{i+1}
\end{array}\right]\left[\begin{array}{cc}
A_{1}^{i} & 0 \\
0 & A_{2}^{i}
\end{array}\right]\left[\begin{array}{cc}
0 & 0 \\
0 & I_{n-r}
\end{array}\right] P^{-1}=P\left[\begin{array}{cc}
0 & 0 \\
0 & \left(B_{4}^{d}\right)^{i+1} A_{2}^{i}
\end{array}\right] P^{-1} .
$$

Hence, we get

$$
\sum_{i=0}^{r-1}\left(B^{d}\right)^{i+1} A^{i} A^{\pi}=\sum_{i=0}^{r-1}\left(B^{d}\right)^{i+1} A^{i} A^{\pi}=P\left[\begin{array}{cc}
0 & 0 \\
0 & \sum_{i=0}^{r-1}\left(B_{4}^{d}\right)^{i+1} A_{2}^{i}
\end{array}\right] P^{-1}=P\left[\begin{array}{cc}
0 & 0 \\
0 & \left(A_{2}+B_{4}\right)^{d}
\end{array}\right] P^{-1} .
$$

Therefore,

$$
\sum_{i=0}^{r-1}\left(B^{d}\right)^{i+1} A^{i} A^{\pi} B W^{d}=P\left[\begin{array}{cc}
0 & 0 \\
0 & \left(A_{2}+B_{4}\right)^{d}
\end{array}\right]\left[\begin{array}{cc}
B_{1} & 0 \\
B_{3} & B_{4}
\end{array}\right]\left[\begin{array}{cc}
\left(A_{1}+B_{1}\right)^{d} & 0 \\
0 & 0
\end{array}\right] P^{-1}=P\left[\begin{array}{cc}
0 & 0 \\
\left(A_{2}+B_{4}\right)^{d} B_{3}\left(A_{1}+B_{1}\right)^{d} & 0
\end{array}\right] P^{-1} .
$$

In a similar way, we get for any $j \in \mathbb{N}$

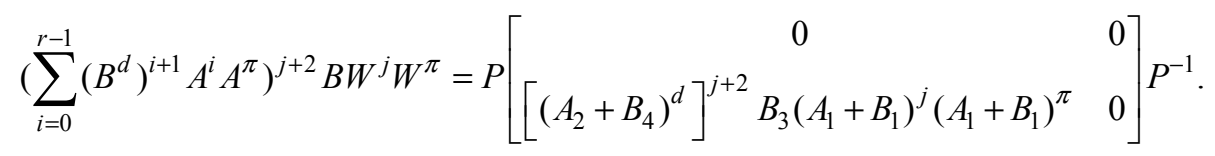

Now, we will find an expression for $\left(A_{2}+B_{4}\right)^{\pi}$. To this end, we use $A_{2} B_{4}=0$ and (3). Observe that 
$A_{2} B_{4}^{d}=A_{2} B_{4}\left(B_{4}^{d}\right)^{2}=0$.

$$
\begin{aligned}
& \left(A_{2}+B_{4}\right)^{\pi}=I_{n-r}-\left(A_{2}+B_{4}\right)\left(A_{2}+B_{4}\right)^{d} \\
= & I_{n-r}-\left(A_{2}+B_{4}\right)\left[B_{4}^{d}+\left(B_{4}^{d}\right)^{2} A_{2}+\left(B_{4}^{d}\right)^{3} A_{2}^{2}+\cdots+\left(B_{4}^{d}\right)^{r} A_{2}^{r-1}\right] \\
= & I_{n-r}-\left[B_{4} B_{4}^{d}+B_{4}\left(B_{4}^{d}\right)^{2} A_{2}+B_{4}\left(B_{4}^{d}\right)^{3} A_{2}^{2}+\cdots+B_{4}\left(B_{4}^{d}\right)^{r} A_{2}^{r-1}\right] \\
= & B_{4}^{\pi}-\left[B_{4}^{d} A_{2}+\left(B_{4}^{d}\right)^{2} A_{2}^{2}+\cdots+\left(B_{4}^{d}\right)^{r-1} A_{2}^{r-1}\right],
\end{aligned}
$$

and so,

$$
\begin{aligned}
\sum_{i=0}^{r-1}\left(B^{d}\right)^{i+1} A^{i+1} A^{\pi}= & B^{d} A A^{\pi}+\left(B^{d}\right)^{2} A^{2} A^{\pi}+\cdots+\left(B^{d}\right)^{r-1} A^{r-1} A^{\pi} \\
& =P\left\{\left[\begin{array}{cc}
0 & 0 \\
0 & B_{4}^{d} A_{2}
\end{array}\right]+\cdots+\left[\begin{array}{cc}
0 & 0 \\
0 & \left(B_{4}^{d}\right)^{r-1} A_{2}^{r-1}
\end{array}\right]\right\} P^{-1} \\
& =P\left[\begin{array}{cc}
0 & 0 \\
0 & B_{4}^{\pi}-\left(A_{2}+B_{4}\right)^{\pi}
\end{array}\right] P^{-1} .
\end{aligned}
$$

In addition,

$$
\begin{aligned}
B^{\pi} & =I-B B^{d} \\
& =P\left\{\left[\begin{array}{cc}
I_{r} & 0 \\
0 & I_{n-r}
\end{array}\right]-\left[\begin{array}{cc}
B_{1} & 0 \\
B_{3} & B_{4}
\end{array}\right]\left[\begin{array}{cc}
B_{1}^{d} & 0 \\
* & B_{4}^{d}
\end{array}\right]\right\} P^{-1} \\
& =P\left[\begin{array}{cc}
I_{r} & 0 \\
* & B_{4}^{\pi}
\end{array}\right] P^{-1},
\end{aligned}
$$

which implies

$$
B^{\pi} A^{\pi}=P\left[\begin{array}{cc}
0 & 0 \\
0 & B_{4}^{\pi}
\end{array}\right] P^{-1} .
$$

Thus,

$$
\begin{aligned}
P\left[\begin{array}{cc}
0 & 0 \\
0 & \left(A_{2}+B_{4}\right)^{\pi}
\end{array}\right] P^{-1} & =P\left[\begin{array}{cc}
0 & 0 \\
0 & B_{4}^{\pi}
\end{array}\right] P^{-1}-\sum_{i=0}^{r-1}\left(B^{d}\right)^{i+1} A^{i+1} A^{\pi} \\
& =B^{\pi} A^{\pi}-\sum_{i=0}^{r-1}\left(B^{d}\right)^{i+1} A^{i+1} A^{\pi} \\
& =\left(B^{\pi}-\sum_{i=0}^{r-1}\left(B^{d}\right)^{i+1} A^{i+1}\right) A^{\pi}
\end{aligned}
$$

Hence for $j \in \mathbb{N}$,

$$
\begin{aligned}
P\left[\begin{array}{cc}
0 & 0 \\
0 & \left(A_{2}+B_{4}\right)^{\pi}\left(A_{2}+B_{4}\right)^{j}
\end{array}\right] P^{-1} & =P\left[\begin{array}{cc}
0 & 0 \\
0 & \left(A_{2}+B_{4}\right)^{\pi}
\end{array}\right]\left[\begin{array}{cc}
0 & 0 \\
0 & \left(A_{2}+B_{4}\right)^{j}
\end{array}\right] P^{-1} \\
& =\left[\left(B^{\pi}-\sum_{i=0}^{r-1}\left(B^{d}\right)^{i+1} A^{i+1}\right) A^{\pi}\right]\left[(A+B)^{j} A^{\pi}\right] .
\end{aligned}
$$

But, as it is easy from (2), one has

$$
A^{\pi}(A+B)^{j} A^{\pi}=(A+B)^{j} A^{\pi} .
$$

Therefore, 


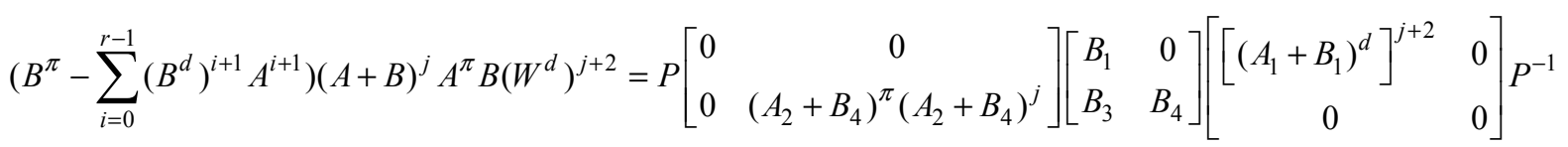

$$
\begin{aligned}
& =P\left[\begin{array}{cc}
0 & 0 \\
\left(A_{2}+B_{4}\right)^{\pi}\left(A_{2}+B_{4}\right)^{j} B_{3}\left[\left(A_{1}+B_{1}\right)^{d}\right]^{j+2} & 0
\end{array}\right] P^{-1} .
\end{aligned}
$$

Finally, let us observe that the expression

$$
\left(\sum_{i=0}^{r-1}\left(B^{d}\right)^{i+1} A^{i} A^{\pi}\right)^{j+2}
$$

in effect, since

$$
\left[\left(A_{2}+B_{4}\right)^{d}\right]^{j+2}=\sum_{i=0}^{r-1}\left(B_{4}^{d}\right)^{i+j+2} A_{2}^{i}
$$

we have that

$$
\left(\sum_{i=0}^{r-1}\left(B^{d}\right)^{i+1} A^{i} A^{\pi}\right)^{j+2}=\sum_{i=0}^{r-1}\left(B^{d}\right)^{i+j+2} A^{i} A^{\pi}
$$

The proof is finished.

The next result, we obtain the same expression for the Drazin inverse $(A+B)^{d}$ as in [15, Theorem2.6] for the Generalized Drazin inverse in a Banach Algebra. Theorem 2.2. Let $A, B \in \mathbb{C}^{n \times n}$ and ind $(A)=r$. If $B A^{\pi}=0$, we have

$$
(A+B)^{d}=W^{d}+A^{\pi} \sum_{i=0}^{r-1} A^{i} B\left(W^{d}\right)^{i+2}
$$

where $W=A A^{d}(A+B)$.

Proof. We can represent $A, A^{d}$ and $B$ as in Theorem 2.1. From $B A^{\pi}=0$, we have

$$
0=B A^{\pi}=P\left[\begin{array}{ll}
B_{1} & B_{2} \\
B_{3} & B_{4}
\end{array}\right]\left[\begin{array}{ll}
0 & 0 \\
0 & I
\end{array}\right] P^{-1}=P\left[\begin{array}{cc}
0 & B_{2} \\
0 & B_{4}
\end{array}\right] P^{-1}
$$

Therefore $B_{2}=B_{4}=0$. Hence,

$$
B=P\left[\begin{array}{ll}
B_{1} & 0 \\
B_{3} & 0
\end{array}\right] P^{-1}, \quad A+B=P\left[\begin{array}{cc}
A_{1}+B_{1} & 0 \\
B_{3} & A_{2}
\end{array}\right] P^{-1}
$$

Observe that

$$
W=A A^{d}(A+B)=P\left[\begin{array}{cc}
A_{1} & 0 \\
0 & A_{2}
\end{array}\right]\left[\begin{array}{cc}
A_{1}^{-1} & 0 \\
0 & 0
\end{array}\right]\left[\begin{array}{cc}
A_{1}+B_{1} & 0 \\
B_{3} & A_{2}
\end{array}\right] P^{-1}=P\left[\begin{array}{cc}
A_{1}+B_{1} & 0 \\
0 & 0
\end{array}\right] P^{-1},
$$

which leads to

$$
W^{d}=P\left[\begin{array}{cc}
\left(A_{1}+B_{1}\right)^{d} & 0 \\
0 & 0
\end{array}\right] P^{-1} .
$$

Thus, by Lemma 2.2, we obtain

$$
(A+B)^{d}=P\left[\begin{array}{cc}
\left(A_{1}+B_{1}\right)^{d} & 0 \\
S & 0
\end{array}\right] P^{-1}
$$


where

$$
S=\sum_{i=0}^{p-1} A_{2}^{i} B_{3}\left[\left(A_{1}+B_{1}\right)^{d}\right]^{i+2},
$$

and $p=\operatorname{ind}\left(A_{2}\right)$.

Also we have

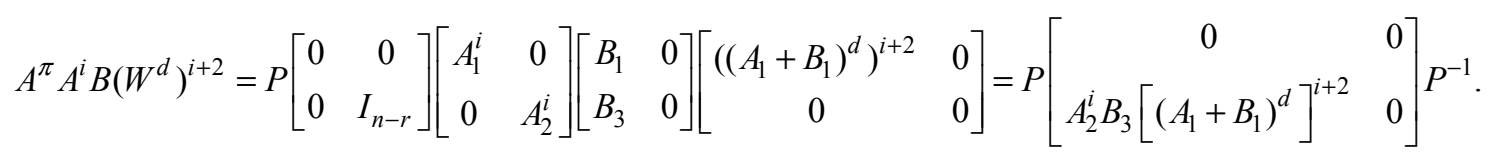

Hence the Theorem follows.

Proof. As in the proof of [5, Theorem7.8.4], we can obtain $(A B)^{d}=A\left((B A)^{d}\right)^{2} B$. The results follow.

\section{Some Results on the Drazin Inverse of $2 \times 2$ Block Matrices}

In this section we shall apply Theorem 2.1 and Theorem 2.2 to obtain some formulas for $M^{d}$ under some conditions when $M$ is a $2 \times 2$ block matrix written as in (1). We assume that ind $(A)=r$, ind $(D)=s$, ind $(B C)=t$, ind $(C B)=l$, and $\max \{\operatorname{ind}(A)$, ind $(D)$, ind $(B C)$, ind $(C B)\}$ $\leq k \leq$ ind $(A)+$ ind $(D)+$ ind $(B C)+$ ind $(C B)$.

Next we will state some auxiliary lemmas.

Lemma $\quad 3.1 \quad$ Let $A \in \mathbb{C}^{m \times n}, B \in \mathbb{C}^{n \times m}$. $A\left((B A)^{d}\right)^{i}=\left((A B)^{d}\right)^{i} A$ for every integer $i \geq 1$, and $B(A B)^{\pi}=(B A)^{\pi} B$. Moreover, ind $(B A)-1 \leq \operatorname{ind}(A B)$ $\leq$ ind $(B A)+1$.
Lemma 3.2 Let $X \in \mathbb{C}^{n \times n}$. Then $\left(X X^{\pi}\right)^{d}=0$, $\left(X^{2} X^{d}\right)^{d}=X^{d},\left(X^{2} X^{d}\right)^{\pi}=X^{\pi}$, and ind $\left(X X^{\pi}\right)=\operatorname{ind}(X)$, ind $\left(X^{2} X^{d}\right)=1$.

Proof. The Jordan canonical form of $X$ permits write $X=S(C \oplus N) S^{-1}$, where $S$ and $C$ are nonsingular, and $N$ is nilpotent. Evidently, $X^{d}=S\left(C^{-1} \oplus 0\right) S^{-1}$. Now, it is evident $X^{2} X^{d}=S(C \oplus 0) S^{-1}$ and $X X^{\pi}=S(0 \oplus N) S^{-1}$, which leads to the affirmations of this lemma.

Lemma 3.3 [10] If $M$ is matrix of a form (1) such that $B C=0$ and $B D=0$, then

$$
M^{d}=\left[\begin{array}{cc}
A^{d} & \left(A^{d}\right)^{2} B \\
\Sigma_{0} & D^{d}+\Sigma_{1} B
\end{array}\right]
$$

where

$$
\Sigma_{n}=\sum_{i=0}^{r-1}\left(D^{d}\right)^{i+n+2} C A^{i} A^{\pi}+D^{\pi} \sum_{i=0}^{s-1} D^{i} C\left(A^{d}\right)^{i+n+2}-\sum_{i=0}^{n}\left(D^{d}\right)^{i+1} C\left(A^{d}\right)^{n-i+1}, n \geq 0 .
$$

Lemma 3.4 [17] Let $M$ be a matrix of a the form (1) with $A=0$ and $D=0$, then

$$
M^{d}=\left[\begin{array}{cc}
0 & B(C B)^{d} \\
(C B) C^{d} & 0
\end{array}\right]
$$

Furthermore, If $\operatorname{ind}(B C)=l$, then $\operatorname{ind}(M) \leq 2 l+1$.

Lemma $3.5[18]$ If $M$ is matrix of a form (1) such that $A B=0$ and $D=0$, then

$$
M^{d}=\left[\begin{array}{cc}
X A & (B C)^{d} B \\
C X & 0
\end{array}\right]
$$

where

$$
X=(B C)^{\pi} \sum_{i=0}^{t-1}(B C)^{i}\left(A^{d}\right)^{2 i+2}+\sum_{i=0}^{\left[\frac{r}{2}\right]-1}\left[(B C)^{d}\right]^{i+1} A^{2 i} A^{\pi}
$$

Using Theorem 2.1 and the previous lemmas, we get the following results. 
Theorem 3.1 Let $M$ be given by (1). If $A B=0$ and $D C A^{\pi}=0$, then

$$
\begin{aligned}
& M^{d}=\left[\begin{array}{cc}
A^{d}+2 X A-R D^{\pi} C A^{d} & R D^{\pi}+(B C)^{d} B D^{\pi} \\
(C B)^{\pi} Z_{0}+C X-S D^{\pi} C A^{d}+C(B C)^{d}\left(A^{\pi}+X A^{2}\right) & (C B)^{\pi} D^{d}+S D^{\pi}
\end{array}\right] \\
& +\left[\begin{array}{cc}
I-B C X & -R D \\
-C X A & (C B)^{\pi}-S D
\end{array}\right]_{j=0}^{k-1}\left[\begin{array}{ll}
A & B \\
C & D
\end{array}\right]^{j}\left[\begin{array}{cc}
B Z_{j+1} & B\left(D^{d}\right)^{j+2} \\
D^{\pi} C\left(A^{d}\right)^{j+2} & 0
\end{array}\right],
\end{aligned}
$$

where

$$
\begin{gathered}
X=\sum_{n=0}^{\left[\frac{r}{2}\right]-1}\left[(B C)^{d}\right]^{i+1} A^{2 i} A^{\pi}, Z_{n}=-\sum_{i=0}^{n}\left(D^{d}\right)^{i+1} C\left(A^{d}\right)^{n-i+1}, \\
R=\sum_{i=0}^{\left[\frac{s}{2}\right]-1}\left[(B C)^{d}\right]^{i+1} B D^{2 i}, S=\sum_{i=0}^{\left[\frac{s}{2}\right]-1}\left[(C B)^{d}\right]^{i+1} D^{2 i+1}
\end{gathered}
$$

Proof. We can split matrix $M$ as $M=P+Q$, where

$$
\begin{gathered}
P=\left[\begin{array}{cc}
A^{2} A^{d} & 0 \\
0 & D
\end{array}\right], Q=\left[\begin{array}{cc}
A A^{\pi} & B \\
C & 0
\end{array}\right], \\
P^{d}=\left[\begin{array}{cc}
A^{d} & 0 \\
0 & D^{d}
\end{array}\right], P^{\pi}=\left[\begin{array}{cc}
A^{\pi} & 0 \\
0 & D^{\pi}
\end{array}\right] .
\end{gathered}
$$

From $A B=0$ and $D C A^{\pi}=0$, we have $P Q P^{\pi}=0$. Applying Theorem 2.1, we get

$$
\begin{aligned}
& M^{d}=W^{d}+\sum_{i=0}^{p-1}\left(Q^{d}\right)^{i+1} P^{i} P^{\pi}-\sum_{i=0}^{p-1}\left(Q^{d}\right)^{i+1} P^{i} P^{\pi} Q W^{d}+\sum_{j=0}^{h-1}\left(\sum_{i=0}^{p-1}\left(Q^{d}\right)^{i+j+2} P^{i}\right) P^{\pi} Q W^{j} W^{\pi}+Q^{\pi} \sum_{j=0}^{h-1}(P+Q)^{j} P^{\pi} Q\left(W^{d}\right)^{j+2} \\
& -\sum_{j=0}^{h-1} \sum_{i=0}^{p-1}\left(Q^{d}\right)^{i+1} P^{i+1}(P+Q)^{j} P^{\pi} Q\left(W^{d}\right)^{j+2}
\end{aligned}
$$

where

$$
\begin{gathered}
\operatorname{ind}(P)=p \text {, ind }(Q)=q, \text { ind }(Q)=q, \\
(\max \{\operatorname{ind}(P), \text { ind }(Q)\} \leq h \leq \operatorname{ind}(P)+\operatorname{ind}(Q), \text { ind }(W) \leq \operatorname{ind}(P)+\operatorname{ind}(Q) .
\end{gathered}
$$

Now we consider the matrices mentioned in the above equation. Clearly, for every integer $i \geq 1$,

$$
P^{2 i}=\left[\begin{array}{cc}
A^{2 i+1} A^{d} & 0 \\
0 & D^{2 i}
\end{array}\right], P^{2 i+1}=\left[\begin{array}{cc}
A^{2 i+1} A^{d} & 0 \\
0 & D^{2 i+1}
\end{array}\right] .
$$

Since $A B=0$, we have $A A^{\pi} B=0$ and matrix Q satisfies the conditions of Lemma 3.5, so we get

$$
Q^{d}=\left[\begin{array}{cc}
X A & (B C)^{d} B \\
C X & 0
\end{array}\right], Q^{\pi}=\left[\begin{array}{cc}
I-B C X & 0 \\
-C X A & (C B)^{\pi}
\end{array}\right],
$$

where $X$ is defined in (6).

From $A B=0$, we have $A X=0, X X=(B C)^{d} X$, 
$X B=(B C)^{d} B, B C(B C)^{d} X=X$ and $X(B C)^{d}=\left((B C)^{d}\right)^{2}$. Then, by Lemma3.1, for every integer $i \geq 1$,

$$
\begin{aligned}
\left(Q^{d}\right)^{2 i} & =\left[\begin{array}{cc}
{\left[(B C)^{d}\right]^{i-1} X} & 0 \\
C\left[(B C)^{d}\right]^{i} X A & {\left[(C B)^{d}\right]^{i}}
\end{array}\right], \\
\left(Q^{d}\right)^{2 i+1} & =\left[\begin{array}{cc}
{\left[(B C)^{d}\right]^{i} X A} & {\left[(B C)^{d}\right]^{i+1} B} \\
C\left[(B C)^{d}\right]^{i} X & 0
\end{array}\right] .
\end{aligned}
$$

From $A B=0$, we have

$$
W=P P^{d}(P+Q)=\left[\begin{array}{cc}
A^{2} A^{d} & A A^{d} B \\
D D^{d} C & D^{2} D^{d}
\end{array}\right]=\left[\begin{array}{cc}
A^{2} A^{d} & 0 \\
D D^{d} C & D^{2} D^{d}
\end{array}\right],
$$

by Lemma 2.2 and Lemma 3.2, we obtain

$$
W^{d}=\left[\begin{array}{cc}
A^{d} & 0 \\
Z_{0} & D^{d}
\end{array}\right],\left(W^{d}\right)^{n}=\left[\begin{array}{cc}
\left(A^{d}\right)^{n} & 0 \\
Z_{n-1} & \left(D^{d}\right)^{n}
\end{array}\right], W^{\pi}=\left[\begin{array}{cc}
A^{\pi} & 0 \\
0 & D^{\pi}
\end{array}\right]
$$

where $Z_{n}$ is defined in (6).

Since $X A A^{\pi}=X A$, we have, for $i=0$,

$$
\sum_{i=0}^{p-1}\left(Q^{d}\right)^{i+1} P^{i} P^{\pi}=Q^{d} P^{\pi}+\left(Q^{d}\right)^{2} P P^{\pi}=\left[\begin{array}{cc}
X A & (B C)^{d} B D^{\pi} \\
C X & (C B)^{d} D D^{\pi}
\end{array}\right],
$$

since $X A A^{d}=0$, we have, for every integer $i \geq 1$,

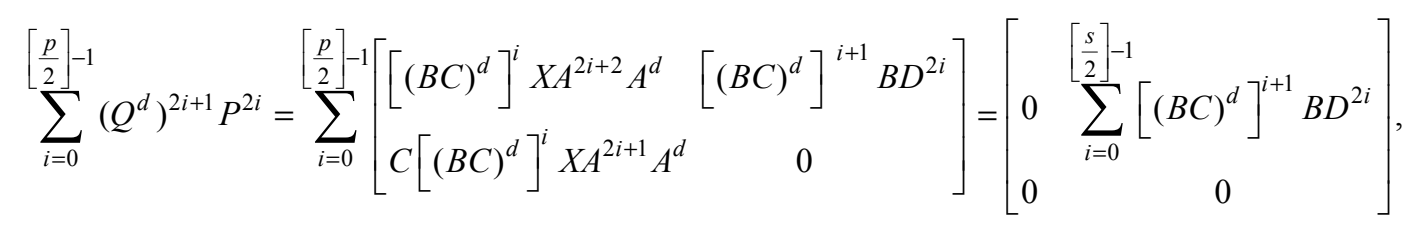

and

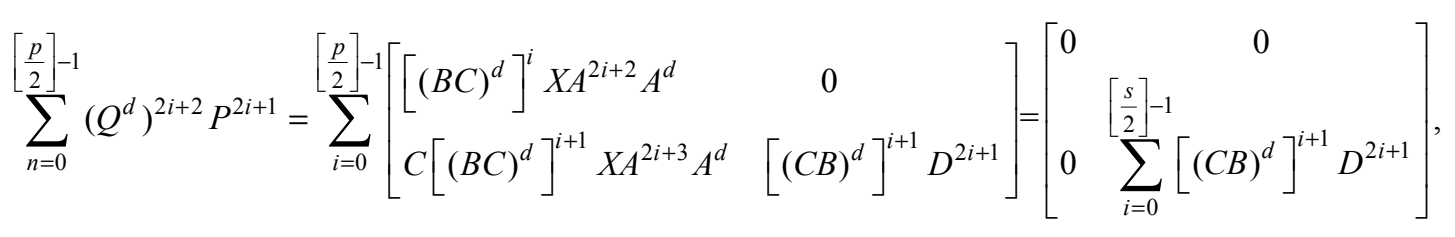

And, similarly

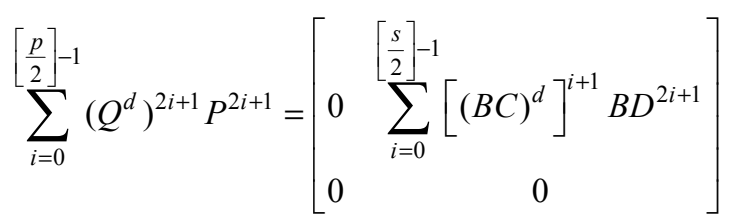

and 


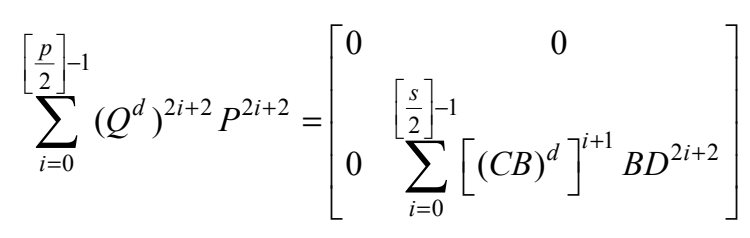

Hence the first sum in (8) is,

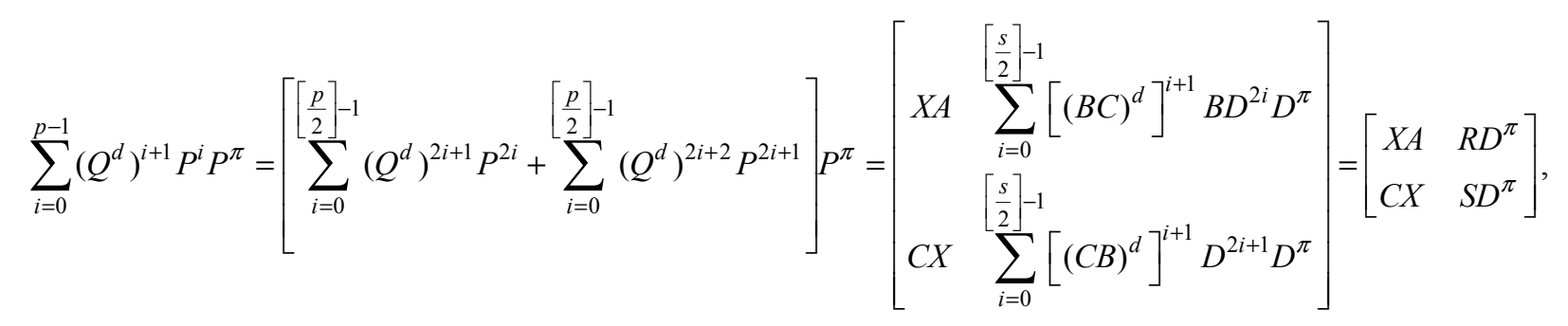

where $R$ and $S$ are defined in (7).

Next consider the second sum in (8).

Note that

$$
Q W^{d}=\left[\begin{array}{cc}
A A^{\pi} & B \\
C & 0
\end{array}\right]\left[\begin{array}{cc}
A^{d} & 0 \\
Z_{0} & D^{d}
\end{array}\right]=\left[\begin{array}{cc}
B Z_{0} & B D^{d} \\
C A^{d} & 0
\end{array}\right]
$$

Since $X B=(B C)^{d} B$ and $A B=0$, using Lemma3.1, we get

$$
\sum_{i=0}^{p-1}\left(Q^{d}\right)^{i+1} P^{i} P^{\pi} Q W^{d}=\left[\begin{array}{cc}
X A & R D^{\pi} \\
C X & S D^{\pi}
\end{array}\right]\left[\begin{array}{cc}
B Z_{0} & B D^{d} \\
C A^{d} & 0
\end{array}\right]=\left[\begin{array}{cc}
R D^{\pi} C A^{d} & 0 \\
C B(C B)^{d} Z_{0}+S D^{\pi} C A^{d} & C B(C B)^{d} D^{d}
\end{array}\right] .
$$

Since $D C A^{\pi}=0$, we have

$$
P P^{\pi} Q W^{\pi}=\left[\begin{array}{cc}
0 & 0 \\
0 & D D^{\pi}
\end{array}\right]\left[\begin{array}{cc}
A A^{\pi} & B \\
C & 0
\end{array}\right]\left[\begin{array}{cc}
A^{\pi} & 0 \\
0 & D^{\pi}
\end{array}\right]=\left[\begin{array}{cc}
0 & 0 \\
D D^{\pi} C A^{\pi} & 0
\end{array}\right]=\left[\begin{array}{ll}
0 & 0 \\
0 & 0
\end{array}\right],
$$

and

$$
W W^{\pi}=\left[\begin{array}{cc}
A^{2} A^{d} & 0 \\
D D^{d} C & D^{2} D^{d}
\end{array}\right]\left[\begin{array}{cc}
A^{\pi} & 0 \\
0 & D^{\pi}
\end{array}\right]=\left[\begin{array}{cc}
A^{2} A^{d} A^{\pi} & 0 \\
D D^{d} C A^{\pi} & D^{2} D^{d} D^{\pi}
\end{array}\right]=\left[\begin{array}{ll}
0 & 0 \\
0 & 0
\end{array}\right] .
$$

Since $X B=(B C)^{d} B, A B=0$ and $D C A^{\pi}=0$, we can prove

$$
\begin{aligned}
& \sum_{j=0}^{h-1}\left(\sum_{i=0}^{p-1}\left(Q^{d}\right)^{i+j+2} P^{i}\right) P^{\pi} Q W^{j} W^{\pi}=\left(Q^{d}\right)^{2} P^{\pi} Q W^{\pi}=\left[\begin{array}{cc}
X & 0 \\
C(B C)^{d} X A & (C B)^{d}
\end{array}\right]\left[\begin{array}{cc}
A A^{\pi} & A^{\pi} B D^{\pi} \\
D^{\pi} C A^{\pi} & 0
\end{array}\right] \\
& =\left[\begin{array}{cc}
X A A^{\pi} & X B D^{\pi} \\
C(B C)^{d} X A^{2} A^{\pi}+(C B)^{d} D^{\pi} C A^{\pi} & C(B C)^{d} X A A^{\pi} B D^{\pi}
\end{array}\right]=\left[\begin{array}{cc}
X A & (B C)^{d} B D^{\pi} \\
C(B C)^{d} X A^{2}+(C B)^{d} C A^{\pi} & 0
\end{array}\right] .
\end{aligned}
$$

Observe that (9) and (10) yield

$$
\sum_{i=0}^{p-1}\left(Q^{d}\right)^{i+1} P^{i+1}=\sum_{i=0}^{\left[\frac{p}{2}\right]-1}\left(Q^{d}\right)^{2 i+1} P^{2 i+1}+\sum_{i=0}^{\left[\frac{p}{2}\right]^{-1}}\left(Q^{d}\right)^{2 i+2} P^{2 i+2}=\left[\begin{array}{cc}
0 & R D \\
0 & S D
\end{array}\right],
$$

So we get 


$$
\begin{aligned}
& Q^{\pi} \sum_{j=0}^{h-1}(P+Q)^{j} P^{\pi} Q\left(W^{d}\right)^{j+2}-\sum_{j=0}^{h-1} \sum_{i=0}^{p-1}\left(Q^{d}\right)^{i+1} P^{i+1}(P+Q)^{j} P^{\pi} Q\left(W^{d}\right)^{j+2}, \\
& =\left[\begin{array}{cc}
I-B C X & -R D \\
-C X A & (C B)^{\pi}-S D
\end{array}\right]_{j=0}^{k-1}\left[\begin{array}{cc}
A & B \\
C & D
\end{array}\right]^{j}\left[\begin{array}{cc}
B Z_{j+1} & B\left(D^{d}\right)^{j+2} \\
D^{\pi} C\left(A^{d}\right)^{j+2} & 0
\end{array}\right] .
\end{aligned}
$$

The proof is finished

If we assume that $A B D^{\pi}=0$ and $D D^{d} C=0$ instead of $\mathrm{AB}=0$, we will get another expression for $M^{d}$.

Theorem 3.2 Let $M$ be given by (1). If $A B D^{\pi}=0, D C A^{\pi}=0$ and $D D^{d} C=0$, then

$$
\left.\begin{array}{l}
M^{d}=\left[\begin{array}{l}
A^{d}+X A+(B C)^{\pi} L-B Y\left(C A^{d}+D S\right) \\
C X-Y D C A^{d}+C(B C)^{d} A^{\pi}+(C B)^{\pi} S-Y D^{2} S
\end{array}\right. \\
Z_{1}-X A\left(B D^{d}+A N\right)+B(C B)^{d} D^{\pi}+(B C)^{\pi} N+B Y\left(I-C Z_{1}-D R\right) \\
D^{d}-C X\left(B D^{d}+A N\right)+(C B)^{\pi} R+Y D\left(I-C Z_{1}-D R\right)
\end{array}\right]
$$

where

$$
\begin{aligned}
& X=\sum_{n=0}^{\left[\frac{r}{2}\right]^{-1}}\left[(B C)^{d}\right]^{n+1} A^{2 n} A^{\pi}, Y=\sum_{n=0}^{\left[\frac{s}{2}\right]-1}\left[(C B)^{d}\right]^{n+1} D^{2 n} D^{\pi}, Z_{n}=-\sum_{i=0}^{n-1}\left(A^{d}\right)^{i+1} B\left(D^{d}\right)^{n-i} \\
& L=\sum_{n=1}^{k-1} B\left(\sum_{i=0}^{n-1}(C B)^{i} D^{2 n-2 i-1} C\left(A^{d}\right)^{2 n+2}+\sum_{i=0}^{n}(C B)^{i} D^{2 n-2 i} C\left(A^{d}\right)^{2 n+3}\right) \\
& N=\sum_{n=1}^{k-1} B\left(\sum_{i=0}^{n-1}(C B)^{i} D^{2 n-2 i-1} C Z_{2 n+2}+\sum_{i=0}^{n}(C B)^{i} D^{2 n-2 i} C Z_{2 n+3} \sum_{i=0}^{n}(B C)^{i} A^{2 n-2 i} A^{\pi}\left(B+A B D^{d}\right)\left(D^{d}\right)^{2 n+2}\right), \\
& S=\sum_{n=1}^{k-1}\left(\sum_{i=0}^{n}(C B)^{i} D^{2 n-2 i}\left(C+D C A^{d}\right)\left(A^{d}\right)^{2 n+2}\right) \\
& R=\sum_{n=1}^{k-1}\left(\sum_{i=0}^{n}(C B)^{i} D^{2 n-2 i}\left(C Z_{2 n+2}+D C Z_{2 n+3}\right)+\sum_{i=0}^{n-1}(C B)^{i} C A^{2 n-2 i-1} A^{\pi} B\left(D^{d}\right)^{2 n+2}+\sum_{i=0}^{n}(C B)^{i} C A^{2 n-2 i} A^{\pi} B\left(D^{d}\right)^{2 n+3}\right)
\end{aligned}
$$

Proof. We can split matrix $M$ as $M=P+Q$, where

$$
P=\left[\begin{array}{cc}
A & 0 \\
0 & D
\end{array}\right], \quad Q=\left[\begin{array}{cc}
0 & B \\
C & 0
\end{array}\right], P^{d}=\left[\begin{array}{cc}
A^{d} & 0 \\
0 & D^{d}
\end{array}\right], \quad P^{\pi}=\left[\begin{array}{cc}
A^{\pi} & 0 \\
0 & D^{\pi}
\end{array}\right] .
$$

From lemma 3.4, we have

$$
Q^{d}=\left[\begin{array}{cc}
0 & B(C B)^{d} \\
(C B)^{d} C & 0
\end{array}\right], Q^{\pi}=\left[\begin{array}{cc}
(B C)^{\pi} & 0 \\
0 & (C B)^{\pi}
\end{array}\right] .
$$

From $A B D^{\pi}=0$ and After the course, there were significant differences in the scores of heart sounds, lung sounds, mixed sound and total score between the two groups $(\mathrm{P}<0.05)$, but there was no significant difference in the scores of bowl sound $(\mathrm{P}>$ $0.05)$.

we have $P Q P^{\pi}=0$. Applying Theorem 2.1, we get 


$$
\begin{aligned}
& M^{d}=W^{d}+\sum_{i=0}^{p-1}\left(Q^{d}\right)^{i+1} P^{i} P^{\pi}-\sum_{i=0}^{p-1}\left(Q^{d}\right)^{i+1} P^{i} P^{\pi} Q W^{d} \\
& +\sum_{j=0}^{h-1}\left(\sum_{i=0}^{p-1}\left(Q^{d}\right)^{i+j+2} P^{i}\right) P^{\pi} Q W^{j} W^{\pi}+Q^{\pi} \sum_{j=0}^{h-1}(P+Q)^{j} P^{\pi} Q\left(W^{d}\right)^{j+2}-\sum_{j=0}^{h-1} \sum_{i=0}^{p-1}\left(Q^{d}\right)^{i+1} P^{i+1}(P+Q)^{j} P^{\pi} Q\left(W^{d}\right)^{j+2},
\end{aligned}
$$

where

$$
\operatorname{ind}(P)=p, \operatorname{ind}(Q)=q, \text { ind }(Q)=q, \max \{\text { ind }(P) \text {,ind }(Q)\} \leq h \leq \operatorname{ind}(P)+\operatorname{ind}(Q) \text { and ind }(W) \leq \text { ind }(P)+\operatorname{ind}(Q) .,
$$

From $D D^{d} C=0$, we obtain

$$
W=P P^{d}(P+Q)=\left[\begin{array}{cc}
A^{2} A^{d} & A A^{d} B \\
D D^{d} C & D^{2} D^{d}
\end{array}\right] .
$$

By Lemma 2.2 and Lemma 3.2, we have

$$
W^{d}=\left[\begin{array}{cc}
A^{d} & Z_{1} \\
0 & D^{d}
\end{array}\right],\left(W^{d}\right)^{n}=\left[\begin{array}{cc}
\left(A^{d}\right)^{n} & Z_{n} \\
0 & \left(D^{d}\right)^{n}
\end{array}\right], W^{\pi}=\left[\begin{array}{cc}
A^{\pi} & 0 \\
0 & D^{\pi}
\end{array}\right],
$$

where $Z_{n}$ is defined in (11).

Clearly, for every integer $n \geq 1$,

$$
\left(Q^{d}\right)^{2 n}=\left[\begin{array}{cc}
\left((B C)^{d}\right)^{n} & 0 \\
0 & \left((C B)^{d}\right)^{n}
\end{array}\right],\left(Q^{d}\right)^{2 n+1}=\left[\begin{array}{cc}
0 & B\left((C B)^{d}\right)^{n+1} \\
\left((C B)^{d}\right)^{n+1} C & 0
\end{array}\right] .
$$

Hence

$$
\begin{aligned}
& \sum_{i=0}^{p-1}\left(Q^{d}\right)^{i+1} P^{i} P^{\pi}=\left[\sum_{i=0}^{\left[\frac{p}{2}\right]^{-1}}\left(Q^{d}\right)^{2 i+1} P^{2 i}+\sum_{i=0}^{\left[\frac{p}{2}\right]-1}\left(Q^{d}\right)^{2 i+2} P^{2 i+1}\right] P^{\pi}
\end{aligned}
$$

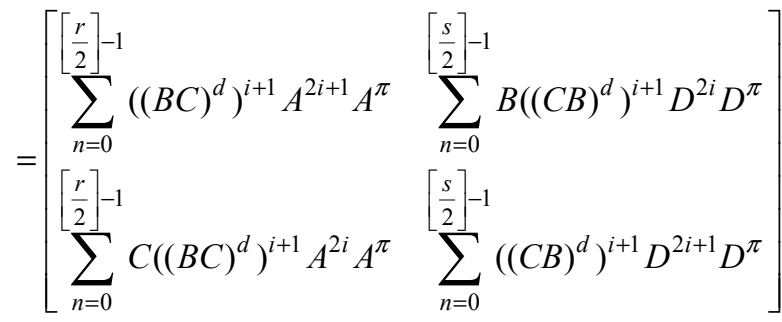

$$
\begin{aligned}
& =\left[\begin{array}{ll}
X A & B Y \\
C X & Y D
\end{array}\right],
\end{aligned}
$$

where $X$ and $Y$ are defined in (11).

$$
Q W^{d}=\left[\begin{array}{cc}
0 & B D^{d} \\
C A^{d} & C Z_{1}
\end{array}\right]
$$

By (16) and (17), we get

$$
\sum_{i=0}^{p-1}\left(Q^{d}\right)^{i+1} P^{i} P^{\pi} Q W^{d}=\left[\begin{array}{cc}
X A & B Y \\
C X & Y D
\end{array}\right]\left[\begin{array}{cc}
0 & B D^{d} \\
C A^{d} & C Z_{1}
\end{array}\right]=\left[\begin{array}{cc}
B Y C A^{d} & X A B D^{d}+B Y C Z_{1} \\
Y D C A^{d} & C X B D^{d}+Y D C Z_{1}
\end{array}\right] .
$$

Since $A B D^{\pi}=0$, we have 


$$
W W^{\pi}=\left[\begin{array}{cc}
A^{2} A^{d} & A A^{d} B \\
0 & D^{2} D^{d}
\end{array}\right]\left[\begin{array}{cc}
A^{\pi} & 0 \\
0 & D^{\pi}
\end{array}\right]=\left[\begin{array}{cc}
A^{2} A^{d} A^{\pi} & A A^{d} B D^{\pi} \\
0 & D^{2} D^{d} D^{\pi}
\end{array}\right]=\left[\begin{array}{ll}
0 & 0 \\
0 & 0
\end{array}\right] .
$$

From $A B D^{\pi}$ and $D C A^{\pi}$, we get

$$
P P^{\pi} Q W^{\pi}=\left[\begin{array}{cc}
0 & A A^{\pi} B D^{\pi} \\
D D^{\pi} C A^{\pi} & 0
\end{array}\right]=\left[\begin{array}{ll}
0 & 0 \\
0 & 0
\end{array}\right]
$$

Since $W^{j} W^{\pi}=0, P P^{\pi} Q W^{\pi}=0, A B D^{\pi}=0$ and $D C A^{\pi}=0$, we have

$$
\begin{aligned}
\sum_{j=0}^{h-1}\left(\sum_{i=0}^{p-1}\left(Q^{d}\right)^{i+j+2} P^{i}\right) P^{\pi} Q W^{j} W^{\pi}= & \left(Q^{d}\right)^{2} P^{\pi} Q W^{\pi} \\
& =\left[\begin{array}{cc}
(B C)^{d} & 0 \\
0 & (C B)^{d}
\end{array}\right]\left[\begin{array}{cc}
A^{\pi} & 0 \\
0 & D^{\pi}
\end{array}\right]\left[\begin{array}{cc}
0 & B \\
C & 0
\end{array}\right]\left[\begin{array}{cc}
A^{\pi} & 0 \\
0 & D^{\pi}
\end{array}\right] \\
& =\left[\begin{array}{cc}
0 & (B C)^{d} A^{\pi} B D^{\pi} \\
(C B)^{d} D^{\pi} C A^{\pi} & 0
\end{array}\right] \\
& =\left[\begin{array}{cc}
0 & (B C)^{d} B D^{\pi} \\
(C B)^{d} C A^{\pi} & 0
\end{array}\right] .
\end{aligned}
$$

The conditions $A B D^{\pi}=0, D C A^{\pi}=0$ and $D D^{d} C=0$ implies that $A B D^{n} C=0$ and $D C B D^{n} C=0$, for $n \geq 0$, observe that (16) and (18) yield

$$
\begin{aligned}
&(P+Q)^{2 i} P^{\pi} Q\left(W^{d}\right)^{2 i+2}= {\left[\begin{array}{ll}
\sum_{i=0}^{n-1} B(C B)^{i} D^{2 n-2 i-1} C\left(A^{d}\right)^{2 n+2} & \sum_{i=0}^{n-1} B(C B)^{i} D^{2 n-2 i-1} C Z_{2 n+2}+\sum_{i=0}^{n}(B C)^{i} A^{2 n-2 i} A^{\pi} B\left(D^{d}\right)^{2 n+2} \\
\sum_{i=0}^{n}(C B)^{i} D^{2 n-2 i} C\left(A^{d}\right)^{2 n+2} & \sum_{i=0}^{n-1}(C B)^{i} D^{2 n-2 i} C Z_{2 n+2}+\sum_{i=0}^{n-1}(C B)^{i} C A^{2 n-2 i-1} A^{\pi} B\left(D^{d}\right)^{2 n+2}
\end{array}\right] } \\
&(P+Q)^{2 n+1} P^{\pi} Q\left(W^{d}\right)^{2 n+3}=\left[\begin{array}{ll}
\sum_{i=0}^{n-1} B(C B)^{i} D^{2 n-2 i} C\left(A^{d}\right)^{2 n+3} & \sum_{i=0}^{n} B(C B)^{i} D^{2 n-2 i} C Z_{2 n+3}+\sum_{i=0}^{n}(B C)^{i} A^{2 n+1-2 i} A^{\pi} B\left(D^{d}\right)^{2 n+3} \\
\sum_{i=0}^{n}(C B)^{i} D^{2 n+1-2 i} C\left(A^{d}\right)^{2 n+3} & \sum_{i=0}^{n}(C B)^{i} D^{2 n+1-2 i} C Z_{2 n+3}+\sum_{i=0}^{n}(C B)^{i} C A^{2 n-2 i} A^{\pi} B\left(D^{d}\right)^{2 n+3}
\end{array}\right] .
\end{aligned}
$$

It follows that

$$
\sum_{n=0}^{h-1}(P+Q)^{n} P^{\pi} Q\left(W^{d}\right)^{n+2}=\left[\left[\sum_{n=0}^{\left[\frac{h}{2}\right]-1}(P+Q)^{2 n} P^{\pi} Q\left(W^{d}\right)^{2 n+2}+\sum_{n=0}^{\left[\frac{h}{2}\right]^{-1}}(P+Q)^{2 n+1} P^{\pi} Q\left(W^{d}\right)^{2 n+3}\right]=\left[\begin{array}{ll}
L & N \\
S & R
\end{array}\right],\right.
$$

where $L, N, S$ and $R$ are defined in (11).

Using the same way as (16), we have

$$
\sum_{n=0}^{p-1}\left(Q^{d}\right)^{n+1} P^{n+1}=\sum_{n=0}^{\left[\frac{p}{2}\right]-1}\left(Q^{d}\right)^{2 n+1} P^{2 n+1}+\sum_{n=0}^{\left[\frac{p}{2}\right]-1}\left(Q^{d}\right)^{2 n+2} P^{2 n+2}=\left[\begin{array}{ll}
X A^{2} & B Y D \\
C X A & Y D^{2}
\end{array}\right] .
$$

The condition $A B D^{n} C=0$ implies that $A L=0$, so we get 
$Q^{\pi} \sum_{j=0}^{h-1}(P+Q)^{j} P^{\pi} Q\left(W^{d}\right)^{j+2}-\sum_{j=0}^{h-1} \sum_{i=0}^{p-1}\left(Q^{d}\right)^{i+1} P^{i+1}(P+Q)^{j} P^{\pi} Q\left(W^{d}\right)^{j+2}=\left[\begin{array}{cc}(B C)^{\pi} L-B Y D S & (B C)^{\pi} N-X A^{2} N-B Y D R \\ (C B)^{\pi} S-Y D^{2} S & (C B)^{\pi} R-C X A N-Y D^{2} R\end{array}\right]$.

The proof is finished.

The next result is a generalization of [9, Theorem 5].

Theorem 3.3 Let $M$ be given by (1), if $D D^{d} C=0$ and $B D^{\pi}=0$. Then

$$
M^{d}=\left[\begin{array}{cc}
A^{d} & -A^{d} B D^{d}+A^{\pi} \sum_{n=0}^{r-1} A^{n} B\left(D^{d}\right)^{n+2} \\
\sum_{n=0}^{s-1} D^{n} C\left(A^{d}\right)^{n+2} & D^{d}+\sum_{n=1}^{s-1} \sum_{i=1}^{n} D^{i-1} C A^{n-i} A^{\pi} B\left(D^{d}\right)^{n+2}+\sum_{n=0}^{s-1} D^{n} C X_{n+2}
\end{array}\right]
$$

where

$$
X_{n}=-\sum_{i=0}^{n-1}\left(A^{d}\right)^{i+1} B\left(D^{d}\right)^{n-i}, \quad n \geq 1 .
$$

Proof. We can split matrix $M$ as $M=P+Q$, where

$$
P=\left[\begin{array}{cc}
A^{2} A^{d} & 0 \\
0 & D^{2} D^{d}
\end{array}\right], \quad Q^{-}\left[\begin{array}{cc}
A A^{\pi} & B \\
C & D D^{\pi}
\end{array}\right], P^{d}=\left[\begin{array}{cc}
A^{d} & 0 \\
0 & D^{d}
\end{array}\right], P^{\pi}=\left[\begin{array}{cc}
A^{\pi} & 0 \\
0 & D^{\pi}
\end{array}\right] .
$$

From $D D^{d} C=0$ and $B D^{\pi}=0$, we have $P Q P^{\pi}=0$. Applying Theorem 2.1, we get

$$
\begin{aligned}
& M^{d}=W^{d}+\sum_{i=0}^{p-1}\left(Q^{d}\right)^{i+1} P^{i} P^{\pi}-\sum_{i=0}^{p-1}\left(Q^{d}\right)^{i+1} P^{i} P^{\pi} Q W^{d}+\sum_{j=0}^{h-1}\left(\sum_{i=0}^{p-1}\left(Q^{d}\right)^{i+j+2} P^{i}\right) P^{\pi} Q W^{j} W^{\pi}+Q^{\pi} \sum_{j=0}^{h-1}(P+Q)^{j} P^{\pi} Q\left(W^{d}\right)^{j+2} \\
& -\sum_{j=0}^{h-1} \sum_{i=0}^{p-1}\left(Q^{d}\right)^{i+1} P^{i+1}(P+Q)^{j} P^{\pi} Q\left(W^{d}\right)^{j+2},
\end{aligned}
$$

where $\operatorname{ind}(P)=p$, ind $(Q)=q$, ind $(Q)=q, \max \{\operatorname{ind}(P)$, ind $(Q)\} \leq h \leq \operatorname{ind}(P)+\operatorname{ind}(Q)$, ind $(W) \leq \operatorname{ind}(P)+$ ind $(Q)$.

Since $D D^{d} C=0$ and $B D^{\pi}=0$, implies, for $n \geq 0, B D^{n} D^{\pi} C=B D^{n} C-B D^{n} D D^{d} C=B D^{n} C=0$.

From $B D^{\pi}=0$ and $B C=0$, the matrix $Q$ satisfies Lemma 3.3, by Lemma3.2, we get

$$
Q^{d}=\left[\begin{array}{cc}
\left(A A^{\pi}\right)^{d} & \left(\left(A A^{\pi}\right)^{d}\right)^{2} B \\
\sum_{0} & \left(D D^{\pi}\right)^{d}+\sum_{1} B
\end{array}\right]=\left[\begin{array}{cc}
0 & 0 \\
\sum_{0} & \Sigma_{1} B
\end{array}\right]
$$

where $\Sigma_{n}$ is defined in (5), since $D D^{d} C=0$, by Lemma3.2, we have $\Sigma_{n}=0$. Thus $Q^{d}=0$ and $Q^{\pi}=I$. In this situation, we obtain $M^{d}=W^{d}+\sum_{j=0}^{h-1}(P+Q)^{j} P^{\pi} Q\left(W^{d}\right)^{j+2}$.

Since $D D^{d} C=0$, we have

$$
W=P P^{d}(P+Q)=\left[\begin{array}{cc}
A^{2} A^{d} & A A^{d} B \\
0 & D^{2} D^{d}
\end{array}\right] .
$$

By Lemma 2.2 and Lemma 3.4, we get 


$$
W^{d}=\left[\begin{array}{cc}
A^{d} & X_{1} \\
0 & D^{d}
\end{array}\right],\left(W^{d}\right)^{n}=\left[\begin{array}{cc}
\left(A^{d}\right)^{n} & X_{n} \\
0 & \left(D^{d}\right)^{n}
\end{array}\right] .
$$

where $X_{n}$ is defined in (19). Clearly, for every integer $n \geq 0$,

$$
P^{\pi} Q\left(W^{d}\right)^{j+2}=\left[\begin{array}{cc}
0 & A^{\pi} B\left(D^{d}\right)^{j+2} \\
C\left(A^{d}\right)^{j+2} & C X_{j+2}
\end{array}\right] .
$$

Since $D D^{d} C=0$ and $B D^{i} C=0$, we can prove, for every integer $n \geq 0$,

$$
\sum_{j=0}^{h-1}(P+Q)^{j} P^{\pi} Q\left(W^{d}\right)^{j+2}=\left[\begin{array}{cc}
0 & \sum_{n=0}^{r-1} A^{\pi} A^{n} B\left(D^{d}\right)^{n+2} \\
\sum_{n=0}^{s-1} D^{n} C\left(A^{d}\right)^{n+2} & \sum_{n=1}^{r-1} \sum_{i=1}^{n} D^{i-1} C A^{n-i} A^{\pi} B\left(D^{d}\right)^{n+2}+\sum_{n=0}^{s-1} D^{n} C X_{n+2}
\end{array}\right]
$$

The proof is finished.

In the rest of the paper we will exploit Theorem 2.2 to obtain some representations of $M^{d}$ under some weaker conditions. Firstly we will present the following result.

Theorem 3.4 Let $M$ be given by (1), if $B D^{\pi}=0, D D^{d} C=0$ and $C A A^{d}=0$. Then

$$
M^{d}=\left[\begin{array}{cc}
A^{d} & X_{1}+\sum_{n=0}^{r-1} A^{\pi} A^{n} B\left(D^{d}\right)^{n+2} \\
0 & D^{d}+\sum_{n=1}^{r-1} \sum_{i=0}^{n-1} D^{n-i-1} C A^{i} B\left(D^{d}\right)^{n+2}
\end{array}\right],
$$

where

$$
X_{n}=-\sum_{i=0}^{n-1}\left(A^{d}\right)^{i+1} B\left(D^{d}\right)^{n-i}, n \geq 1
$$

Proof. We can split matrix $M$ as $M=P+Q$, where

$$
P=\left[\begin{array}{ll}
A & 0 \\
C & D
\end{array}\right], Q=\left[\begin{array}{ll}
0 & B \\
0 & 0
\end{array}\right] .
$$

From $D D^{d} C=0$ and $C A A^{d}=0$, by Lemma 2.2, we get

$$
P^{d}=\left[\begin{array}{cc}
A^{d} & 0 \\
0 & D^{d}
\end{array}\right], \quad P^{\pi}=\left[\begin{array}{cc}
A^{\pi} & 0 \\
0 & D^{\pi}
\end{array}\right] .
$$

Since $D D^{d} C=0$, we have, for every integer $n \geq 1$,

$$
P^{\pi} P^{n} Q=\left[\begin{array}{cc}
0 & A^{\pi} A^{n} B \\
0 & \sum_{i=0}^{n-1} D^{n-i-1} C A^{i} B
\end{array}\right] .
$$

Since $B D^{\pi}=0$, we obtain $Q P^{\pi}=0$. Applying Theorem 2.2, we get

$$
M^{d}=W^{d}+P^{\pi} \sum_{n=0}^{p-1} P^{n} Q\left(W^{d}\right)^{n+2}
$$


From $D D^{d} C=0$ and $C A A^{d}=0$, we have

$$
W=P P^{d}(P+Q)=\left[\begin{array}{cc}
A^{2} A^{d} & A A^{d} B \\
D D^{d} C & D^{2} D^{d}
\end{array}\right]=\left[\begin{array}{cc}
A^{2} A^{d} & A A^{d} B \\
0 & D^{2} D^{d}
\end{array}\right] .
$$

Using the same way as Theorem 3.2, we obtain

$$
W^{d}=\left[\begin{array}{cc}
A^{d} & X_{1} \\
0 & D^{d}
\end{array}\right],\left(W^{d}\right)^{n}=\left[\begin{array}{cc}
\left(A^{d}\right)^{n} & X_{n} \\
0 & \left(D^{d}\right)^{n}
\end{array}\right]
$$

where $X_{n}$ is defined in (20).

Hence, we get

$$
P^{\pi} Q\left(W^{d}\right)^{2}=\left[\begin{array}{cc}
0 & A^{\pi} B \\
0 & 0
\end{array}\right]\left[\begin{array}{cc}
\left(A^{d}\right)^{2} & X_{2} \\
0 & \left(D^{d}\right)^{2}
\end{array}\right]=\left[\begin{array}{cc}
0 & A^{\pi} B\left(D^{d}\right)^{2} \\
0 & 0
\end{array}\right],
$$

apparently

$$
P^{\pi} \sum_{n=1}^{p-1} P^{n} Q\left(W^{d}\right)^{n+2}=\sum_{n=1}^{p-1}\left[\begin{array}{cc}
0 & A^{\pi} A^{n} B \\
0 & \sum_{i=0}^{n-1} D^{n-i-1} C A^{i} B
\end{array}\right]\left[\begin{array}{cc}
\left(A^{d}\right)^{n+2} & X_{n+2} \\
0 & \left(D^{d}\right)^{n+2}
\end{array}\right]=\left[\begin{array}{cc}
0 & \sum_{n=1}^{r-1} A^{\pi} A^{n} B\left(D^{d}\right)^{n+2} \\
0 & \sum_{n=1}^{r-1} \sum_{i=0}^{n-1} D^{n-i-1} C A^{i} B\left(D^{d}\right)^{n+2}
\end{array}\right] .
$$

The proof is finished.

The conditions $B D^{\pi}=0$ and $C A^{\pi}=0$ is less general than $A B D^{\pi}=0$ and $D C A^{\pi}=0$. The following expression for $M^{d}$ is much more simpler than the Theorem3.2.

Theorem 3.5 Let $M$ be given by (1), if $B D^{\pi}=0, D D^{d} C=0$ and $C A^{\pi}=0$. Then

$$
M^{d}=\left[\begin{array}{cc}
A^{d} & X_{1}+\sum_{n=0}^{r-1} A^{\pi} A^{n} B\left(D^{d}\right)^{n+2} \\
\sum_{n=0}^{s-1} D^{n} C\left(A^{d}\right)^{n+2} & D^{d}+\sum_{n=0}^{s-1} D^{n} C X_{n+2}
\end{array}\right]
$$

where

$$
X_{n}=-\sum_{i=0}^{n-1}\left(A^{d}\right)^{i+1} B\left(D^{d}\right)^{n-i}, n \geq 1
$$

Proof. We can represent $P, P^{d}, P^{\pi}$ and $Q$ as in (12). From $D D^{d} C=0$, we have, for $n \geq 0$,

$$
P^{\pi} P^{n} Q=\left[\begin{array}{cc}
0 & A^{\pi} A^{n} B \\
D^{n} C & 0
\end{array}\right]
$$

Since $B D^{\pi}=0$ and $C A^{\pi}=0$, we obtain $Q P^{\pi}=0$. Applying Theorem 2.2, we get

$$
M^{d}=W^{d}+P^{\pi} \sum_{n=0}^{p-1} P^{n} Q\left(W^{d}\right)^{n+2} .
$$

From $D D^{d} C=0$, we have 


$$
W=P P^{d}(P+Q)=\left[\begin{array}{cc}
A^{2} A^{d} & A A^{d} B \\
D D^{d} C & D^{2} D^{d}
\end{array}\right]=\left[\begin{array}{cc}
A^{2} A^{d} & A A^{d} B \\
0 & D^{2} D^{d}
\end{array}\right] .
$$

Using the same way as Theorem 3.2, we have

$$
W^{d}=\left[\begin{array}{cc}
A^{d} & X_{1} \\
0 & D^{d}
\end{array}\right],\left(W^{d}\right)^{n}=\left[\begin{array}{cc}
\left(A^{d}\right)^{n} & X_{n} \\
0 & \left(D^{d}\right)^{n}
\end{array}\right],
$$

where $X_{n}$ is defined in (21).

Hence, we get

$$
\begin{aligned}
& P^{\pi} \sum_{n=0}^{p-1} P^{n} Q\left(W^{d}\right)^{n+2} \\
& =\sum_{n=0}^{p-1}\left[\begin{array}{ccc}
0 & A^{\pi} A^{n} B \\
D^{n} C & 0
\end{array}\right]\left[\begin{array}{cc}
\left(A^{d}\right)^{n+2} & X_{n+2} \\
0 & \left(D^{d}\right)^{n+2}
\end{array}\right] \\
& =\left[\begin{array}{cc}
0 & \sum_{n=0}^{r-1} A^{\pi} A^{n} B\left(D^{d}\right)^{n+2} \\
\sum_{n=0}^{s-1} D^{n} C\left(A^{d}\right)^{n+2} & \sum_{n=0}^{s-1} D^{n} C X_{n+2}
\end{array}\right] .
\end{aligned}
$$

The proof is finished.

Theorem 3.6. Let $M$ be given by (1). If $B D^{\pi}=0, A=B D^{d} C$ and $D^{d} C B=0$. Then

$$
M^{d}=\left[\begin{array}{lc}
B\left(D^{d}\right)^{3} C & B\left(D^{d}\right)^{2} \\
\left(D^{d}\right)^{2} C+\sum_{n=1}^{s-1} D^{n-1} C B\left(D^{d}\right)^{n+3} C & D^{d}+\sum_{n=1}^{s-1} D^{n-1} C B\left(D^{d}\right)^{n+2}
\end{array}\right] .
$$

Proof. We can split matrix $M$ as $M=P+Q$, where

$$
P=\left[\begin{array}{ll}
0 & 0 \\
C & D
\end{array}\right], Q=\left[\begin{array}{ll}
A & B \\
0 & 0
\end{array}\right]
$$

By Lemma 2.2, we have

$$
P^{d}=\left[\begin{array}{cc}
0 & 0 \\
\left(D^{d}\right)^{2} C & D^{d}
\end{array}\right], P^{\pi}=\left[\begin{array}{cc}
I & 0 \\
-D^{d} C & D^{\pi}
\end{array}\right] .
$$

From $B D^{\pi}=0, A=B D^{d} C$ and $D^{d} C B=0$, we have $A B=B D^{d} C B=0, D^{d} C A=D^{d} C B D^{d} C=0$.

From $D^{d} C A=0$ and $D^{d} C B=0$, we have, for $n \geq 1$,

$$
P^{\pi} P^{n} Q=\left[\begin{array}{cc}
0 & 0 \\
D^{n-1} C A & D^{n-1} C B
\end{array}\right] .
$$

Since $B D^{\pi}=0$ and $A=B D^{d} C$, we obtain $Q P^{\pi}=0$. Applying Theorem 2.2, we get

$$
M^{d}=W^{d}+P^{\pi} \sum_{n=0}^{p-1} P^{n} Q\left(W^{d}\right)^{n+2} .
$$

From $D^{d} C A=0$ and $D^{d} C B=0$, we have 


$$
\begin{aligned}
W & =P P^{d}(P+Q) \\
& =\left[\begin{array}{cc}
0 & 0 \\
D^{d} C & D D^{d}
\end{array}\right]\left[\begin{array}{ll}
A & B \\
C & D
\end{array}\right] \\
& =\left[\begin{array}{cc}
0 & 0 \\
D^{d} C A+D D^{d} C & D^{d} C B+D^{2} D^{d}
\end{array}\right] \\
& =\left[\begin{array}{cc}
0 & 0 \\
D D^{d} C & D^{2} D^{d}
\end{array}\right],
\end{aligned}
$$

By Lemma 2.2 and Lemma 3.2, we have

$$
W^{d}=\left[\begin{array}{cc}
0 & 0 \\
\left(D^{d}\right)^{2} C & D^{d}
\end{array}\right],\left(W^{d}\right)^{n}=\left[\begin{array}{cc}
0 & 0 \\
\left(D^{d}\right)^{n+1} C & \left(D^{d}\right)^{n}
\end{array}\right] .
$$

The conditions $D^{d} C A=0$ and $D^{d} C B=0$ implies that $P^{\pi} Q=Q$. Hence, we get

$$
P^{\pi} Q\left(W^{d}\right)^{2}=\left[\begin{array}{ll}
A & B \\
0 & 0
\end{array}\right]\left[\begin{array}{cc}
0 & 0 \\
\left(D^{d}\right)^{3} C & \left(D^{d}\right)^{2}
\end{array}\right]=\left[\begin{array}{cc}
B\left(D^{d}\right)^{3} C & B\left(D^{d}\right)^{2} \\
0 & 0
\end{array}\right]
$$

and

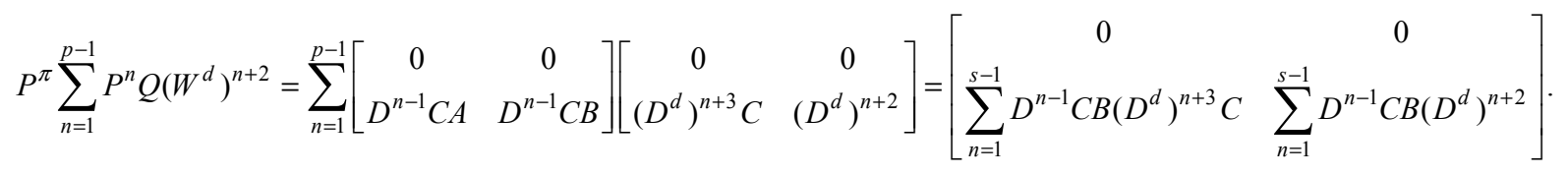

The proof is finished.

Theorem 3.6 has the following dual version.

$$
M^{d}=\left[\begin{array}{c}
A^{d}+\sum_{n=1}^{r-1} A^{n-1} B C\left(A^{d}\right)^{n+2} \\
C\left(A^{d}\right)^{2}
\end{array}\right.
$$

Proof. Using the same way as Theorem 3.6, we can obtain the result.

\section{Acknowledgements}

The authors are very grateful to the reviewers for their suggestions. This research was supported by Guangxi Natural Science Foundation (No. 2016GXNSFAA380125).

\section{References}

[1] M. P. Drazin, Pseudo-inverses in associative rings and semiproup, Amer. Math. Monthly, 65 (1958), 506-514.

[2] R. E. Harte, Invertibility and Singularity, Dekker, 1988.

[3] S. L. Campbell, The Drazin inverse and systerms of second order linear differential equations, linear Multilinear Algebra, 14 (1983), 195-198.

[4] S. L. Campbell, C. D. Meyer Jr., N. J. Rose, Applications of the Drazin inverse to linear systerms of differential equations, SIAM J. Apll. Math., 31 (1976), 411-425.
Theorem 3.7 Let $M$ be given by (1). If $C A^{\pi}=0$, $D=C A^{d} B$ and $A^{d} B C=0$. Then

$$
\left.\begin{array}{c}
\left(A^{d}\right)^{2} B+\sum_{n=1}^{r-1} A^{n-1} B C\left(A^{d}\right)^{n+3} B \\
C\left(A^{d}\right)^{3} B
\end{array}\right]
$$

[5] S. L. Campbell, C. D. Meyer, Generalized Inverse of Linear Trasformations, Pitman, London, 1979, Dover, New York, 1991.

[6] N. Zhang, Y. Wei, Sloving EP singular linear systems, Int. J. Comput. Math., 81 (2004), 1395-1405.

[7] C. D. Meyer Jr., N. J. Rose, The index and the Drazin inverse of block triangular matrices, SIAM J. Appl. Math. 33 (1) (1977), $1-7$.

[8] R. E. Hartwig, J. M. Shoaf, Gruop inverse and Drazin inverse of bidiagonal and triangular toeplitz matrices, Austral. J. Math., 24 (A) (1977), 10-34.

[9] G. Yan, X. Qin, X. Liu, Representations for the Drazin inverse of block matrices, Alabama Journal of Mathematics, 41 (2017): $1-10$.

[10] E. Dopazo, M. F. Martínez-Serrano, Further results on the representation of the Drazin inverse of a $2 \times 2$ block matrices, Linear Algebra Appl., 432 (2010), 1896-1904.

[11] D. S. Cvetkovi'c-Ili'c, A note on the representation for the Drazin inverse of $2 \times 2$ blockmatrices. Linear Algebra Appl., 429 (2008), 242-248. 
[12] D. Mosi'c, A note on the representations for the generalized Drazin inverse of block matrices, Acta Mathematica Scientia. 35B (2015): 1483-1491.

[13] H. Yang, X. Liu, The Drazin inverse of the sum of two matrices and its applications, J. Comput. Appl. Math., 235 (2011), 1412-1417.

[14] L. Xia, B. Deng, The Drazin Inverse of the Sum of Two Matrices and its Applications, Filomat, 16 (2017), 5151-5158.

[15] X. Liu, X. Qin, J. Benítez, New additive results for the generalized Drazin inverse in a Banach algebra, Filomat, 30 (2016), 2289-2294
[16] R. E. Hartwig, G. R. Wang, and Y. Wei, Some additive results on Drazin inverse, Linear Algebra Appl., 322 (2001), 207-217.

[17] M. Catral, D. D. Olesky, and P. Driessche, Block representations of the Drazin inverse of a bipartite block matrix, Linear Algebra Appl. 5 A (09) (2009), 98-107.

[18] C. Deng, Y. Wei, A note on the Drazin inverse of an anti-triangular matrix, Linear Algebra Appl., 431 (2009), 1910-1922. 